\title{
WKB approximation for inflationary cosmological perturbations
}

\author{
Jérôme Martin* \\ Institut d'Astrophysique de Paris, 98bis boulevard Arago, 75014 Paris, France \\ Dominik J. Schwarz ${ }^{\dagger}$ \\ Theory Division, CERN, 1211 Geneva 23, Switzerland \\ and Institut für Theoretische Physik, Technische Universität Wien, Wiedner Hauptstraße 8-10, 1040 Vienna, Austria
}

(Received 4 October 2002; published 24 April 2003)

\begin{abstract}
A new method for predicting inflationary cosmological perturbations, based on the Wentzel-KramersBrillouin (WKB) approximation, is presented. A general expression for the WKB scalar and tensor power spectra is derived. The main advantage of the new scheme of approximation is that it is valid even if the slow-roll conditions are violated. The method is applied to power-law inflation, which allows a comparison with an exact result. It is demonstrated that the WKB approximation predicts the spectral indices exactly and the amplitude with an error lower than $10 \%$, even in regimes far from scale invariance. The new method of approximation is also applied to a situation where the slow-roll conditions hold. It is shown that the result obtained bears close resemblance with the standard slow-roll calculation. Finally, some possible improvements are briefly mentioned.
\end{abstract}

DOI: 10.1103/PhysRevD.67.083512

PACS number(s): 98.80.Cq, 98.70.Vc

\section{INTRODUCTION}

Observations of the inhomogeneities and anisotropies of the Universe's large scale structure and of the cosmic microwave background (CMB) radiation are one of the keys to decipher the cosmic evolution. According to the favored cosmological scenario, fluctuations of matter and space-time are generated during an early epoch of inflation. The ability to predict the power spectra of cosmological perturbations for a large variety of different inflationary models is of the utmost importance. So far, all predictions that come up to the level of accuracy needed for CMB experiments such as Archeops, the Microwave Anisotropy Probe (MAP) or Planck, have to rely on the slow-roll approximation, subject to an infinite number of convergence conditions, or on numerical integration [1]. In this paper we present a new method that overcomes the mentioned restrictions (but has its own, very different ones). Our method is applicable to models that cannot be described by the slow-roll approximation (e.g. the "problem of a large slow-roll parameter $\eta$ " of some supersymmetry-inspired inflationary models) and it can be applied to small scales that leave the horizon at times close to the end of inflation, where the slow-roll approximation necessarily has to break down.

The study of scalar and tensor fluctuations can be reduced to the study of a single variable during inflation, usually denoted by $\mu_{\mathrm{S}}$ or $\mu_{\mathrm{T}}$ [2]. The corresponding equations of motion are similar to the Schrödinger equation for a onedimensional system:

$$
\frac{\mathrm{d}^{2}}{\mathrm{~d} \eta^{2}} \mu+\left[k^{2}-U(\eta)\right] \mu=\frac{\mathrm{d}^{2}}{\mathrm{~d} \eta^{2}} \mu+\omega^{2}(\eta) \mu=0
$$

\footnotetext{
*Electronic address: jmartin@iap.fr

†Electronic address: dominik.schwarz@cern.ch
}

where, in the cosmological context, the conformal time $\eta$ plays the role of the radial variable $r$ in atomic physics. In general, this equation cannot be solved exactly unless very simple potentials $U(\eta)$ are considered and one has to rely on a method of approximation. However, we have inherited a very powerful method to deal with more complicated potentials from the pioneers of quantum mechanics, namely the Wentzel-Kramers-Brillouin (WKB) method of approximation.

In this article, we apply this method to the equation governing the evolution of cosmological perturbations. It is quite astonishing that for more than twenty years since the discovery of cosmological inflation, to our knowledge, nobody has been able to apply successfully the WKB approximation to the prediction of cosmological perturbations. This is probably related to the fact that the growth of the perturbations on superhorizon scales, which is equivalent, in the quantummechanical version of the theory, to particles creation, is often described in terms of the breakdown of the WKB approximation. In this article, we demonstrate that this is true only if the WKB approximation is used naively. In fact, the problem bears a close resemblance with the situation discussed by atomic physicists at the time quantum mechanics was born. We therefore make a historical digression to illustrate the main point of the present article.

The subject debated by the atomic physicists at the beginning of the twentieth century was the application of the WKB approximation to the motion in a central field of force and, more specifically, how the Balmer formula, for the energy levels of hydrogenic atoms, can be recovered within the WKB approximation. The effective frequency for hydrogenic atoms is given by (obviously, in the atomic physics context, the wave equation is not a differential equation with respect to time but to the radial coordinate $r$ )

$$
\omega^{2}(r)=\frac{2 m}{\hbar^{2}}\left(E+\frac{Z e^{2}}{r}\right)-\frac{\ell(\ell+1)}{r^{2}},
$$


where $Z e$ is the (attractive) central charge and $\ell$ the quantum number of angular momentum. The symbol $E$ denotes the energy of the particle and is negative in the case of a bound state. The calculation of the energy levels was first addressed by Kramers [3] and by Young and Uhlenbeck [4]. The method employed was Sommerfeld's quantization rule

$$
\oint \omega(r) \mathrm{d} r=2 \pi\left(n+\frac{1}{2}\right)
$$

where the integral must be calculated along the classical trajectory given here by an ellipse. The result obtained by those authors reads

$$
E_{n, \ell}=-\frac{m Z^{2} e^{4}}{2 \hbar^{2}}\left\{\left(n+\frac{1}{2}\right)+[\ell(\ell+1)]^{1 / 2}\right\}^{-2} .
$$

In addition, the WKB wave function for small values of $r$ was found to behave as $\simeq r^{[\ell(\ell+1)]^{1 / 2}+1 / 2}$. This last result follows straightforwardly if one retains only the last term in Eq. (2), i.e. $\omega(r) \simeq i[\ell(\ell+1)]^{1 / 2} / r$, as required in the limit $r \rightarrow 0$. It was noticed by Young and Uhlenbeck that the two previous results are not satisfactory. Indeed, experimental results indicate that the factor $\ell(\ell+1)$ in Eq. (4) should be replaced by $(\ell+1 / 2)^{2}$ : "It is apparent that we do not obtain the familiar Balmer formula except in the limit of large $\ell$ values. If, however, we replace $\ell(\ell+1)$ by $(\ell+1 / 2)^{2}$ the correct result is obtained." A similar remark is made in Ref. [4] concerning the behavior of the wave function near the origin: "The approximate solution based upon the negative exponential behaves for $r$ very small like $r^{[\ell(\ell+1)]^{1 / 2}+1 / 2}$. Again the replacement of $\ell(\ell+1)$ by $(\ell+1 / 2)^{2}$ leads to an agreement between the approximate and exact solutions." The solution advocated by Young and Uhlenbeck was therefore to replace, by hand, $\ell(\ell+1)$ by $(\ell+1 / 2)^{2}$ without further theoretical justifications.

In 1937 the problem was considered again by Langer [5]. In the section devoted to the radial wave equation, he notices that the WKB approximation was applied to the Schrödinger equation (1) by Kramers, Young and Uhlenbeck only because of its resemblance with the equation of an oscillator: "Insofar as the writer is aware, the degree of this resemblance has hitherto been regarded without exception as sufficient for assuming the out-and-out applicability to the equation (17) of the formulas deduced in the foregoing discussion, especially of the formula (14)," where Eqs. (17) and (14) in Langer's paper correspond to Eq. (1) and the WKB connection formula. He also remarks that no theoretical explanation has been given to explain why the wrong factor $\ell(\ell+1)$ is obtained: "This 'failure' of the WKB method, i.e. to the extent that the change in question is requisite, has been generally verified both in studies of attractive and repulsive fields. No explanation of it seems to have been given, though that can be done very simply to the following effect. The fault lies not in the method but in the application of it." Then, Langer shows that the WKB approximation breaks down, at small $r$, for an effective frequency given by Eq. (2) and, in addition, he suggests a method to circumvent this difficulty. The method consists in changing the variables according to

$$
r=\mathrm{e}^{x}, \quad \mu=\mathrm{e}^{x / 2} u .
$$

The resulting equation for $u$ has again the form of Eq. (1) but the effective frequency is now given by

$$
\omega^{2}(x)=\frac{2 m}{\hbar^{2}}\left(E \mathrm{e}^{2 x}+Z e^{2} \mathrm{e}^{x}\right)-\left(\ell+\frac{1}{2}\right)^{2} .
$$

As demonstrated by Langer, the crucial point is that, now, the WKB approximation, applied to the Schrödinger equation for $u(x)$, no longer breaks down. Therefore, it is now possible to utilize the WKB formalism and to use the usual WKB approximation. Transforming back to the original variables the correct factor $(\ell+1 / 2)^{2}$ appears, instead of the wrong term $[\ell(\ell+1)]^{1 / 2}$. This allows Langer to conclude that his method " $\ldots$ is seen to amount formally to precisely the replacement of $\ell(\ell+1)$ by $(\ell+1 / 2)^{2}$."

Let us now come back to the theory of cosmological perturbations. The analogy with the previous situation is that large values of $r$ correspond to subhorizon scales $(k|\eta| \gg 1)$ whereas small values of $r$ correspond to superhorizon scales $(k|\eta| \ll 1)$. On subhorizon scales the mode function oscillates $(\omega \simeq k)$ and the WKB approximation can be applied in this regime without any difficulty. On superhorizon scales the perturbations do not oscillate, but this is not a problem in itself. The WKB approximation can be used in a situation where the potential energy dominates and leads to exponentially decaying or growing solutions (e.g. in the WKB treatment of the $\alpha$ decay of a radioactive nucleus). Nevertheless, difficulties arise if one tries to find the WKB approximation to the mode equations of cosmological perturbations.

On superhorizon scales a problem arises in the naive WKB approach in the manner of Kramers, Uhlenbeck and Young from the form of the effective potential $U(\eta)$. It turns out to be the very same problem as encountered by atomic physicists. The resemblance is most explicit in the particular case of power-law inflation where the potential $U(\eta) \propto \eta^{-2}$ is exactly of the form given in Eq. (2). The usual conclusion that the WKB approximation is violated for superhorizon scales is in fact not true, provided the cosmological version of the transformation (5) can be found. This opens the possibility to employ the WKB approximation to calculate the spectrum of inflationary cosmological perturbations in the superhorizon limit. In the cosmological context, the WKB approximation is a new method for computing the power spectra, different from the more traditional slow-roll approximation.

This article is organized as follows. In the next section, we briefly recall the basic results of the inflationary cosmological perturbations theory. In the third section, we show how the WKB approximation can be applied to inflationary cosmological perturbations in the general case, i.e. for any inflaton potential. The only restriction is that we focus on single inflaton field models. We demonstrate that the calculation of the spectrum reduces to the calculation of a single 
quadrature. In the fourth section we apply the WKB approximation to power-law inflation. This model is exactly solvable so that we can compare the exact result with the WKB prediction and also with the standard calculation based on the slow-roll approximation. This allows us to study the precision of the WKB approximation in the cosmological context. In particular, we demonstrate that, in the regime where the slow-roll approximation breaks down (far from scale invariance), the WKB approximation is still valid. This opens the possibility to calculate the power spectra of more realistic models violating the slow-roll conditions. In the fifth section, we apply the WKB approximation to the general (first-order) slow-roll situation. We compare the result with the standard slow-roll calculation for the amplitudes and the spectral indices. We show that the spectral indices are very well predicted while the error in the amplitude is of the order $10 \%$, i.e. less accurate than the prediction of the slow-roll approximation for this quantity. Finally, in the sixth section, we give our conclusions and indicate what the possible extensions to the present work are.

\section{GENERAL EXPRESSIONS FOR INFLATIONARY PERTURBATIONS}

\section{A. Power spectra}

The evolution of the cosmological perturbations in a single field inflationary scenario is governed by the Schrödinger-like equation (1). The effective time-dependent frequency is given by the general expression

$$
\omega^{2}(k, \eta)=k^{2}-\frac{z^{\prime \prime}}{z},
$$

where the primes denote a derivatives with respect to conformal time and where $z_{\mathrm{s}}=a \sqrt{-a a^{\prime \prime}} / a^{\prime}$ for scalar (density) perturbations and $z_{\mathrm{T}}=a$ for tensor perturbations; $a(\eta)$ is the scale factor of the Friedmann-Lemaitre-Robertson-Walker metric. For density perturbations, the quantity $\mu$ is given by $\mu_{\mathrm{s}}=-2 z_{\mathrm{s}} \zeta$, where $\zeta$ is Bardeen's hypersurface-independent quantity [6], which for adiabatic perturbations is conserved on superhorizon scales. For tensor fluctuations, one simply has $\mu_{\mathrm{T}}=z_{\mathrm{T}} h$, where $h$ is the amplitude of gravitational waves. The equation of motion for the mode functions $\mu$ should be solved with the following initial condition

$$
\lim _{k /(a H) \rightarrow+\infty} \mu_{\mathrm{S}, \mathrm{T}}(\eta)=\mp \frac{4 \sqrt{\pi}}{m_{\mathrm{Pl}}} \frac{\mathrm{e}^{-i k\left(\eta-\eta_{\mathrm{i}}\right)}}{\sqrt{2 k}},
$$

where $\eta_{\mathrm{i}}$ is an arbitrary time at the beginning of inflation and $m_{\mathrm{Pl}}$ is the Planck mass. This initial condition corresponds to the fact that, initially, the modes are subhorizon and, therefore, do not feel the curvature of space-time. As a consequence, they are described by plane waves. If the initial quantum state is the vacuum state, then the statistical properties of the perturbations are entirely characterized by the two-point correlation function, i.e. by the power spectrum. The dimensionless power spectra of scalar and tensor fluctuations are calculated to read

$$
\mathcal{P}_{\zeta}=\frac{k^{3}}{8 \pi^{2}}\left|\frac{\mu_{\mathrm{S}}}{z_{\mathrm{S}}}\right|^{2}, \quad \mathcal{P}_{h}=\frac{2 k^{3}}{\pi^{2}}\left|\frac{\mu_{\mathrm{T}}}{z_{\mathrm{T}}}\right|^{2} .
$$

The spectral indices and their running are defined by the coefficients of Taylor expansions of the power spectra with respect to $\ln k$, evaluated at an arbitrary pivot scale $k_{*}$. To be more specific:

$$
n_{\mathrm{S}}-1 \equiv\left|\frac{\mathrm{d} \ln \mathcal{P}_{\zeta}}{\mathrm{d} \ln k}\right|_{k=k_{*}},\left.\quad n_{\mathrm{T}} \equiv \frac{\mathrm{d} \ln \mathcal{P}_{h}}{\mathrm{~d} \ln k}\right|_{k=k_{*}},
$$

are the spectral indices and the two following expressions

$$
\left.\alpha_{\mathrm{S}} \equiv \frac{\mathrm{d}^{2} \ln \mathcal{P}_{\zeta}}{(\mathrm{d} \ln k)^{2}}\right|_{k=k_{*}},\left.\quad \alpha_{\mathrm{T}} \equiv \frac{\mathrm{d}^{2} \ln \mathcal{P}_{h}}{(\mathrm{~d} \ln k)^{2}}\right|_{k=k_{*}},
$$

define the "running" of these indices. In principle, we could also define the running of the running and so on.

\section{B. Inflationary parameters}

In order to investigate the predictions of cosmological inflation in a model-independent manner, it is useful to define a set of functions that uniquely characterize the state of the Universe at any given moment of time. It is useful to describe the evolution of the Universe by a set of flow equations $[7,8]$. In this article we make use of the set of horizon flow functions, introduced in Ref. [8]. The zeroth horizon flow function is defined by $\epsilon_{0} \equiv H\left(N_{\mathrm{i}}\right) / H(N)$, where $H$ $\equiv \dot{a} / a$ is the Hubble rate, a dot meaning derivative with respect to cosmic time. In this expression, $N$ is the number of e-folds, $N \equiv \ln \left(a / a_{\mathrm{i}}\right)$, after an arbitrary initial time. The hierarchy of horizon flow functions is then defined according to

$$
\epsilon_{n+1} \equiv \frac{\mathrm{d} \ln \left|\epsilon_{n}\right|}{\mathrm{d} N}, \quad n \geqslant 0 .
$$

Inflation takes place for $\epsilon_{1}<1$. It is interesting to establish the link between the horizon flow functions and the so-called slow-roll parameters, the first three being defined by

$$
\begin{aligned}
& \epsilon \equiv 3 \frac{\dot{\varphi}^{2}}{2}\left[\frac{\dot{\varphi}^{2}}{2}+V(\varphi)\right]^{-1}=-\frac{\dot{H}}{H^{2}}, \\
& \delta \equiv-\frac{\ddot{\varphi}}{H \dot{\varphi}}=-\frac{\dot{\epsilon}}{2 H \epsilon}+\epsilon, \\
& \xi \equiv \frac{\dot{\epsilon}-\dot{\delta}}{H}
\end{aligned}
$$

where $\varphi$ is the inflaton field and $V(\varphi)$ is the inflaton potential. The first two slow-roll parameters must be small during inflation, as can be seen from their definition. The parameter $\epsilon$ is just the inflaton kinetic energy to inflaton total energy ratio, whereas $\delta$ is the acceleration to speed ratio. The set $\{\epsilon, \delta, \xi\}$ is linked to the horizon flow functions $\left\{\epsilon_{n}\right\}$ by 


$$
\epsilon=\epsilon_{1}, \quad \delta=\epsilon_{1}-\frac{1}{2} \epsilon_{2}, \quad \xi=\frac{1}{2} \epsilon_{2} \epsilon_{3} .
$$

The set $\{\epsilon, \delta, \xi\}$ or equivalently the set $\left\{\epsilon_{1}, \epsilon_{2}, \epsilon_{3}\right\}$ plays a special role, since the effective potential $U(\eta) \equiv z^{\prime \prime} / z$ of Eq. (1) can be expressed exactly in terms of these three functions only. One finds

$$
\begin{aligned}
& U_{\mathrm{S}}=a^{2} H^{2}\left(2-\epsilon_{1}+\frac{3}{2} \epsilon_{2}-\frac{1}{2} \epsilon_{1} \epsilon_{2}+\frac{1}{4} \epsilon_{2}^{2}+\frac{1}{2} \epsilon_{2} \epsilon_{3}\right), \\
& U_{\mathrm{T}}=a^{2} H^{2}\left(2-\epsilon_{1}\right) .
\end{aligned}
$$

Let us emphasize that these expressions are exact, no approximation has been used so far.

\section{WKB POWER SPECTRA}

We now turn to the derivation of the WKB inflationary power spectra for scalar and tensor perturbations. We define the quantity $\mu_{\mathrm{WKB}}$ by the expression

$$
\mu_{\mathrm{WKB}}(k, \eta) \equiv \frac{1}{\omega^{1 / 2}(k, \eta)} \mathrm{e}^{ \pm i \int \eta(k, \tau) \mathrm{d} \tau} .
$$

The mode function $\mu_{\mathrm{WKB}}$ represents the leading order term of the semiclassical expansion. It satisfies the following differential equation

$$
\mu_{\mathrm{WKB}}^{\prime \prime}(k, \eta)+\left[\omega^{2}(k, \eta)-Q(k, \eta)\right] \mu_{\mathrm{WKB}}(k, \eta)=0,
$$

where the quantity $Q(k, \eta)$ is given by

$$
Q(k, \eta) \equiv \frac{3}{4} \frac{\left(\omega^{\prime}\right)^{2}}{\omega^{2}}-\frac{\omega^{\prime \prime}}{2 \omega} .
$$

Therefore, the mode function $\mu_{\mathrm{WKB}}(k, \eta)$ given in Eq. (19) is a good approximation of the actual mode function $\mu(k, \eta)$, if the following condition is satisfied:

$$
\left|\frac{Q}{\omega^{2}}\right| \ll 1
$$

On subhorizon scales, $\omega \simeq k$, which implies $Q \simeq 0$ and therefore the condition is satisfied. On superhorizon scales, it is not possible to show that the previous condition is always violated, since it depends on the shape of the effective potential $U(\eta)$. However, in the cosmological context, the problem discussed in the Introduction generally arises. We explicitly show this in the next section for the case of powerlaw inflation. Here, we demonstrate the problem for the more generic case of slow-roll inflation. Using the expression for the effective frequency in terms of the horizon flow functions, one has

$$
\left|\frac{Q}{\omega^{2}}\right|_{\mathrm{S}}=\left|\frac{Q}{\omega^{2}}\right|_{\mathrm{T}}=\frac{1}{8}+\mathcal{O}\left(\epsilon_{1}, \epsilon_{2}\right) .
$$

The corrections, linear in the horizon flow functions, could easily multiply the term $1 / 8=0.125$ by a factor 2 . Therefore, the number $\left|Q / \omega^{2}\right|$ is indeed not greater than 1 but, at the same time, not very small with respect to 1 . Thus, the WKB approximation is not a good approximation in this regime. In order to have an effective potential that permits to use the WKB approximation, we introduce the following transformation:

$$
x \equiv \ln \left(\frac{H a}{k}\right), \quad u \equiv\left(1-\epsilon_{1}\right)^{1 / 2} \mathrm{e}^{x / 2} \mu .
$$

This is the cosmological counterpart of Langer's transformation given in the Introduction; see Eq. (5). However, let us remark that there exist differences, in particular the factor $\left(1-\epsilon_{1}\right)^{1 / 2}$ in the definition of the function $u$. It is also interesting to notice that this transformation has already been considered in the literature in Ref. [9] for different reasons. Then, for density perturbations, the equation of motion takes the form

$$
\begin{gathered}
\frac{\mathrm{d}^{2} u_{\mathrm{S}}(x)}{\mathrm{d} x^{2}}+\left[\frac{\mathrm{e}^{-2 x}}{\left(1-\epsilon_{1}\right)^{2}}-\frac{1}{4}\left(\frac{3-\epsilon_{1}}{1-\epsilon_{1}}\right)^{2}-\frac{\left(3-2 \epsilon_{1}\right) \epsilon_{2}}{2\left(1-\epsilon_{1}\right)^{2}}\right. \\
\left.-\frac{\left(1-2 \epsilon_{1}\right) \epsilon_{2} \epsilon_{3}}{2\left(1-\epsilon_{1}\right)^{3}}-\frac{\left(1-4 \epsilon_{1}\right) \epsilon_{2}^{2}}{4\left(1-\epsilon_{1}\right)^{4}}\right] u_{\mathrm{S}}(x)=0,
\end{gathered}
$$

whereas, for gravitational waves, we obtain

$$
\begin{gathered}
\frac{\mathrm{d}^{2} u_{\mathrm{T}}(x)}{\mathrm{d} x^{2}}+\left[\frac{\mathrm{e}^{-2 x}}{\left(1-\epsilon_{1}\right)^{2}}-\frac{1}{4}\left(\frac{3-\epsilon_{1}}{1-\epsilon_{1}}\right)^{2}+\frac{\epsilon_{1} \epsilon_{2}}{2\left(1-\epsilon_{1}\right)^{2}}\right. \\
\left.+\frac{\epsilon_{1} \epsilon_{2} \epsilon_{3}}{2\left(1-\epsilon_{1}\right)^{3}}+\frac{\left(2+\epsilon_{1}\right) \epsilon_{1} \epsilon_{2}^{2}}{4\left(1-\epsilon_{1}\right)^{4}}\right] u_{\mathrm{T}}(x)=0 .
\end{gathered}
$$

In the following $\omega_{\mathrm{S}}^{2}$ and $\omega_{\mathrm{T}}^{2}$ denote the expressions in the square brackets of Eqs. (25) and (26). The WKB approximation can now be applied to these equations. In order to demonstrate this, let us recalculate, on superhorizon scales, the quantity $\left|Q / \omega^{2}\right|$ for the two previous expressions. One finds for density perturbations

$$
\left|\frac{Q}{\omega^{2}}\right|_{\mathrm{S}}=\frac{4}{27}\left(\epsilon_{1} \epsilon_{2}^{2}+\epsilon_{1} \epsilon_{2} \epsilon_{3}+\frac{\epsilon_{2} \epsilon_{3}^{2}}{2}+\frac{\epsilon_{2} \epsilon_{3} \epsilon_{4}}{2}\right)+\mathcal{O}\left(\epsilon_{n}^{4}\right)
$$

whereas for gravitational waves, one has

$$
\left|\frac{Q}{\omega^{2}}\right|_{\mathrm{T}}=\frac{4}{27}\left(\epsilon_{1} \epsilon_{2}^{2}+\epsilon_{1} \epsilon_{2} \epsilon_{3}\right)+\mathcal{O}\left(\epsilon_{n}^{4}\right) .
$$


It is obvious that the quantity $\left|Q / \omega^{2}\right|$ is now very small for any inflaton potential satisfying the slow-roll conditions, since this is a third-order quantity. As a consequence, the WKB approximation is now also valid on superhorizon scales.

Let us apply the WKB approximation to Eqs. (25) and (26). Let $x_{*}$ be the value of the variable $x$ such that $\omega^{2}\left(x_{*}\right)=0$, i.e. $x_{*}$ is the classical turning point. We define region $\mathrm{I}$ as the region such that $\omega^{2}(x)>0$ and region II the region where $\omega^{2}(x)<0$. Let $x_{\mathrm{i}}$ be the initial "time" at which the normalization is performed (i.e. corresponding to $\eta_{\mathrm{i}}$ ). At some point $x$, deep in region $\mathrm{I}$, the WKB approximation of the function $u(x)$ can be written as

$$
u_{\mathrm{I}}(x) \simeq \frac{A}{\omega^{1 / 2}(x)} \exp \left[ \pm i \int_{x_{\mathrm{i}}}^{x} \omega(y) \mathrm{d} y\right]
$$

where $A$ is a constant of normalization. Deep in region I, we have $\omega(y) \simeq \mathrm{e}^{-y} /\left(1-\epsilon_{1}\right)$ and straightforward manipulations show that the corresponding $\mu_{\mathrm{I}}(\eta)$ behaves as in Eq. (8), provided that one chooses the minus sign in Eq. (29) and that $A$ is given by

$$
A=\mp \frac{4}{m_{\mathrm{Pl}}} \sqrt{\frac{\pi}{2}} k^{-1 / 2} .
$$

In region II, the effective frequency is complex and can be written as $\omega(x)=i|\omega(x)|$. Let $x_{\mathrm{f}}$ be the final point at which we evaluate the solution deep in region II. Then, at any point $x$ in region II, the WKB solution is given by

$$
\begin{aligned}
u_{\mathrm{II}}(x) \simeq & \frac{C_{+}}{|\omega(x)|^{1 / 2}} \exp \left[+\int_{x}^{x_{\mathrm{f}}}|\omega(y)| \mathrm{d} y\right] \\
& +\frac{C_{-}}{|\omega(x)|^{1 / 2}} \exp \left[-\int_{x}^{x_{\mathrm{f}}}|\omega(y)| \mathrm{d} y\right],
\end{aligned}
$$

where $C_{ \pm}$are constant. The goal is now to connect these two constants with the constant $A$. At the turning point $x=x_{*}$, the WKB approximation breaks down and one has to use the usual WKB procedure and approximate the potential in this region by a straight line such that

$$
\omega^{2}(x) \simeq-\alpha\left(x-x_{*}\right),
$$

where $\alpha \equiv-\left[\mathrm{d} \omega^{2}(x) / \mathrm{d} x\right]\left(x=x_{*}\right)>0$. The solutions of Eqs. (25) and (26) are given in terms of Airy functions of first and second kinds

$$
u(x)=B_{1} \operatorname{Ai}(s)+B_{2} \operatorname{Bi}(s)
$$

where $s \equiv \alpha^{1 / 3}\left(x-x_{*}\right)$.

The next move is to use the asymptotic behavior of the Airy functions to calculate the relation between $B_{1}$ and $B_{2}$ with $A$ on one hand and $C_{ \pm}$on the other. In region I, for a value of $x$ not too far from $x_{*}$, Eq. (33) can be written as

$$
\begin{aligned}
u_{\mathrm{I}}(x) \simeq & \frac{\alpha^{-1 / 12}}{2 \sqrt{\pi}}\left|x-x_{*}\right|^{-1 / 4}\left[( B _ { 2 } - i B _ { 1 } ) \operatorname { e x p } \left(\frac{i 2}{3} \alpha^{1 / 2}\left|x-x_{*}\right|^{3 / 2}\right.\right. \\
& \left.\left.+\frac{i \pi}{4}\right)+\left(B_{2}+i B_{1}\right) \exp \left(-\frac{i 2}{3} \alpha^{1 / 2}\left|x-x_{*}\right|^{3 / 2}-\frac{i \pi}{4}\right)\right],
\end{aligned}
$$

whereas in region II, under the same conditions, the function $u(x)$ can be expressed as

$$
\begin{aligned}
u_{\mathrm{II}}(x) \simeq & \frac{\alpha^{-1 / 12}}{\sqrt{\pi}}\left(x-x_{*}\right)^{-1 / 4}\left\{\frac{B_{1}}{2} \exp \left[-\frac{2}{3} \alpha^{1 / 2}\left(x-x_{*}\right)^{3 / 2}\right]\right. \\
& \left.+B_{2} \exp \left[\frac{2}{3} \alpha^{1 / 2}\left(x-x_{*}\right)^{3 / 2}\right]\right\} .
\end{aligned}
$$

There is no factor $1 / 2$ in front of the coefficient $B_{2}$ because the expansion of the function Bi differs from the expansion of Ai not only in the sign of the argument of the exponential but also by a factor $1 / 2$. Now we evaluate in region I, the approximate solution (29) for the potential (32). The integral in the exponent can be written as

$$
\int_{x_{\mathrm{i}}}^{x_{*}} \omega(y) \mathrm{d} y=\int_{x_{\mathrm{i}}}^{x} \omega(y) \mathrm{d} y+\int_{x_{*}}^{x} \omega(y) \mathrm{d} y \equiv \Phi+\int_{x_{*}}^{x} \omega(y) \mathrm{d} y .
$$

The frequency (32) is used in the second integral only, assuming that $x$ is not too far away from $x_{*}$. The quantity $\Phi$ is just a number and its calculation would require the knowledge of $\omega(x)$ in the whole region I. However, it does not enter the final result and therefore we are not interested in its value. We find

$$
u_{\mathrm{I}}(x)=A \alpha^{-1 / 4}\left|x-x_{*}\right|^{-1 / 4} \exp \left(\frac{i 2}{3} \alpha^{1 / 2}\left|x-x_{*}\right|^{3 / 2}+i \Phi\right),
$$

from which we deduce

$$
B_{1}=i B_{2}, \quad B_{2}=A \sqrt{\pi} \alpha^{-1 / 6} e^{i(\Phi-\pi / 4)} .
$$

Therefore, we have reached our first goal, i.e. connect the two coefficients $B_{1}$ and $B_{2}$ to $A$. What remains to be done is to establish the link between $C_{+}, C_{-}$and $B_{1}, B_{2}$. This can be done by applying the very same reasoning in region II. One introduces $\Psi$, defined by

$$
\Psi \equiv \int_{x_{*}}^{x_{\mathrm{f}}}|\omega(y)| \mathrm{d} y,
$$

and the WKB solution given in Eq. (31) reduces to the expression

$$
\begin{aligned}
u_{\mathrm{II}}(x)= & \alpha^{-1 / 4}\left(x-x_{*}\right)^{-1 / 4}\left\{C_{+} e^{\Psi} \exp \left[-\frac{2}{3} \alpha^{1 / 2}\left(x-x_{*}\right)^{3 / 2}\right]\right. \\
& \left.+C_{-} e^{-\Psi} \exp \left[\frac{2}{3} \alpha^{1 / 2}\left(x-x_{*}\right)^{3 / 2}\right]\right\} .
\end{aligned}
$$


By comparison with Eq. (35), the expressions for $C_{+}$and $C_{\text {- }}$ are obtained

$$
C_{+}=\frac{B_{2}}{2 \sqrt{\pi}} \alpha^{1 / 6} \mathrm{e}^{-\Psi+i \pi / 2}, \quad C_{-}=\frac{B_{2}}{\sqrt{\pi}} \alpha^{1 / 6} \mathrm{e}^{\Psi} .
$$

Finally, putting everything together, we arrive at

$$
\left|C_{+}\right|^{2}=\frac{2 \pi}{m_{\mathrm{Pl}}^{2}} \mathrm{e}^{-2 \Psi} k^{-1}, \quad\left|C_{-}\right|^{2}=\frac{8 \pi}{m_{\mathrm{Pl}}^{2}} \mathrm{e}^{2 \Psi} k^{-1}
$$

If we only consider the growing mode given by the branch proportional to $C_{-}$, which is a good approximation for $x$ $\gg x_{*}$, one can deduce the power spectra at $x=x_{\mathrm{f}}$. Using Eqs. (9), one obtains the dimensionless power spectra within the WKB approximation:

$$
\begin{aligned}
& \mathcal{P}_{\zeta}=\frac{H^{2}}{\pi \epsilon_{1} m_{\mathrm{Pl}}^{2}}\left(\frac{k}{a H}\right)^{3} \frac{\mathrm{e}^{2 \Psi_{\mathrm{S}}}}{\left(1-\epsilon_{1}\right)\left|\omega_{\mathrm{S}}\right|}, \\
& \mathcal{P}_{h}=\frac{16 H^{2}}{\pi m_{\mathrm{Pl}}^{2}}\left(\frac{k}{a H}\right)^{3} \frac{\mathrm{e}^{2 \Psi_{\mathrm{T}}}}{\left(1-\epsilon_{1}\right)\left|\omega_{\mathrm{T}}\right|},
\end{aligned}
$$

where all the quantities in the above expression are evaluated at $x=x_{\mathrm{f}}$. We know that in the absence of entropy perturbations and decaying modes, $\zeta$ is conserved on superhorizon scales [6,11]. Similarly, $h$ is conserved on superhorizon scales, if the decaying mode is absent. Thus we know that both spectra should be time-independent. However, within the WKB approximation one cannot see this from the above expressions. In the following sections we demonstrate this property explicitly for power-law inflation and slow-roll inflation.

We see that the calculation of the spectrum for any inflaton potential has been reduced to the calculation of a quadrature, namely $\Psi$. We are also in a position to calculate the spectral indices and their running. The spectral indices read

$$
n_{\mathrm{S}}-1=3+\left.2 \frac{\mathrm{d} \Psi_{\mathrm{S}}}{\mathrm{d} \ln k}\right|_{k=k_{*}}, \quad n_{\mathrm{T}}=3+\left.2 \frac{\mathrm{d} \Psi_{\mathrm{T}}}{\mathrm{d} \ln k}\right|_{k=k_{*}},
$$

and the runnings are given by

$$
\alpha_{\mathrm{S}}=\left.2 \frac{\mathrm{d}^{2} \Psi_{\mathrm{S}}}{(\mathrm{d} \ln k)^{2}}\right|_{k=k_{*}}, \quad \alpha_{\mathrm{T}}=\left.2 \frac{\mathrm{d}^{2} \Psi_{\mathrm{T}}}{(\mathrm{d} \ln k)^{2}}\right|_{k=k_{*}} .
$$

The general expressions for the WKB power spectra, spectral indices and their runnings, constitute the main result of this work. In order to discuss the power and potential of the WKB approximation, we now turn to two cases where the quadratures $\Psi_{\mathrm{S}}$ and $\Psi_{\mathrm{T}}$ can be calculated explicitly: powerlaw and slow-roll inflation.

\section{APPLICATION TO POWER-LAW INFLATION}

We first apply the formalism developed in the previous section to power-law inflation. In this model, the scale factor is given by

$$
a(\eta)=\ell_{0}|\eta|^{1+\beta},
$$

where $\beta \leqslant-2$. This scale factor is a solution of the Einstein equations in the case where the inflaton potential is $V$ $\propto \exp \left(\varphi / m_{\mathrm{PI}}\right)$. The case $\beta=-2$ is special, since it corresponds to the de Sitter space-time. The Hubble radius is then constant and given by $\ell_{0}$. In the general case, the horizon flow functions parameters are easily calculated and read

$$
\epsilon_{1}=\frac{2+\beta}{1+\beta}, \quad \epsilon_{n}=0, n>1 .
$$

The exact power spectrum can be found and is given by $[10,11]$

$$
\begin{aligned}
& \mathcal{P}_{\zeta}=\frac{\ell_{\mathrm{Pl}}^{2}}{\ell_{0}^{2}} \frac{1}{\pi \epsilon_{1}} f(\beta) k^{2 \beta+4}, \\
& \mathcal{P}_{h}=\frac{\ell_{\mathrm{Pl}}^{2}}{\ell_{0}^{2}} \frac{16}{\pi} f(\beta) k^{2 \beta+4},
\end{aligned}
$$

where $\ell_{\mathrm{Pl}}=m_{\mathrm{Pl}}^{-1}$ is the Planck length and the function $f(\beta)$ is given by

$$
f(\beta) \equiv \frac{1}{\pi}\left[\frac{\Gamma(-\beta-1 / 2)}{2^{\beta+1}}\right]^{2} .
$$

In the previous expression, $\Gamma$ denotes Euler's integral of the second kind. We have $f(\beta=-2)=1$. The case $\beta=-2$ is singular, since $\epsilon_{1}=0$ and the expression of the scalar power spectrum blows up. This case should be considered separately and one can show that there are no density perturbations at all in the de Sitter space-time [11]. The spectral indices and runnings can also be calculated very easily. One finds $n_{\mathrm{S}}-1=n_{\mathrm{T}}=2 \beta+4$ and $\alpha_{\mathrm{S}}=\alpha_{\mathrm{T}}=0$. Exact scale invariance is obtained for $\beta=-2$, which is precisely the singular case evoked above.

Let us now apply the WKB approximation to the case under consideration. First, suppose that we want to use it before doing the transformation (24). In this case, we have $\omega^{2}(\eta)=k^{2}-\beta(\beta+1) / \eta^{2}$. In the limit where $k \eta$ goes to zero, it is easy to see that

$$
\left|\frac{Q}{\omega^{2}}\right|=\frac{1}{4|\beta(\beta+1)|} .
$$

In general the condition $\left|Q / \omega^{2}\right| \ll 1$ is not satisfied unless $-\beta$ goes to infinity, an unrealistic situation. For $\beta=-2$, one recovers that $\left|Q / \omega^{2}\right|=1 / 8$. This is in agreement with the calculation of $\left|Q / \omega^{2}\right|$ performed previously in the slow-roll approximation since this approximation is, very roughly speaking, an expansion around the scale-invariant solution. 
Equation (52) shows that the WKB approximation cannot be applied to the usual equation of motion without first performing the transformation (24). This conclusion is reinforced by the following argument. The solution on superhorizon scales is exactly known and can be expressed as

$$
\mu_{\mathrm{II}}(\eta)=C_{1} z(\eta)+C_{2} z(\eta) \int^{\eta} \frac{\mathrm{d} \tau}{z^{2}(\tau)},
$$

where $C_{1}$ and $C_{2}$ are two arbitrary constants. The first branch is the growing mode whereas the second one is the decaying mode. For power-law inflation this gives a mode proportional to $\mu \propto|\eta|^{1+\beta}$ and one proportional to $\mu$ $\propto|\eta|^{-\beta}$. One can wonder what the link between this solution and the WKB solution is in region II. On superhorizon scales, we have $|\omega| \simeq[\beta(\beta+1)]^{1 / 2} /|\eta|$. As a consequence, the mode function $\mu_{\mathrm{WKB}}$ has the form $\mu_{\mathrm{WKB}}$ $\propto|\eta|^{1 / 2 \pm[\beta(\beta+1)]^{1 / 2}}$. This is not the correct behavior given by the previous exact solution. The correct solution is obtained by replacing the factor $\beta(\beta+1)$ by $(\beta+1 / 2)^{2}$, i.e. exactly the remark made by Kramers, Young and Uhlenbeck (see the Introduction). The reason is very clear: the effective powerlaw potential has exactly the same shape as the one describing the motion in a central-force field, provided that the identification $\ell \leftrightarrow \beta$ is made. Let us also remark that the form of $\mu_{\text {WКB }}$ is the same as the wave function for small $r$, as expected from the previous considerations. This confirms that the WKB approximation cannot be used naively on superhorizon scales.
The cure is to use the transformation given in Eq. (24). After this, the effective frequency takes the form

$$
\omega_{\mathrm{S}}^{2}(x)=\omega_{\mathrm{T}}^{2}(x)=(1+\beta)^{2} \mathrm{e}^{-2 x}-\left(\beta+\frac{1}{2}\right)^{2} .
$$

In the superhorizon limit, $x \rightarrow \infty$, one now has $Q \rightarrow 0$, indicating that the WKB solution gives a good approximation. In addition, the mode function now has the correct time dependence. Indeed, since $\int_{x}^{x_{\mathrm{f}}}|\omega| \mathrm{d} y=|\beta+1 / 2|\left(x_{\mathrm{f}}-x\right)$, returning to the dependence in conformal time gives $u_{\mathrm{II}} \propto|\eta|^{ \pm(\beta+1 / 2)}$. Taking into account the link between $u$ and $\mu$, one finally arrives at $\mu_{\mathrm{II}} \propto|\eta|^{1+\beta}$ or $\mu_{\mathrm{II}} \propto|\eta|^{-\beta}$, i.e. the growing and decaying modes of Eq. (53) in the case of power-law inflation. This means that, in this case, the superhorizon solution is nothing but the WKB solution. However, this need not be true for more general models. A change of variables, which allows us to come back from $x$ to the conformal time $\eta$, shows that the quantity $\Psi$ is given by

$$
\Psi=-\int_{\mathrm{k} \eta_{\mathrm{f}}}^{k \eta_{*}} \frac{\mathrm{d}(k \eta)}{k \eta} \sqrt{-k^{2} \eta^{2}+\left(\beta+\frac{1}{2}\right)^{2}}
$$

where $\eta_{*}$ can now be expressed as $k \eta_{*}=\beta+1 / 2$. This integral can be performed exactly, for instance with the help of formula (2.275.3) of Ref. [12]. One obtains

$$
\begin{aligned}
\Psi & =-\left\{\sqrt{-k^{2} \eta_{\mathrm{f}}^{2}+\left(\beta+\frac{1}{2}\right)^{2}}+\frac{1}{2}\left|\beta+\frac{1}{2}\right| \ln \left[\frac{|\beta+1 / 2|-\sqrt{-k^{2} \eta_{\mathrm{f}}^{2}+(\beta+1 / 2)^{2}}}{|\beta+1 / 2|+\sqrt{-k^{2} \eta_{\mathrm{f}}^{2}+(\beta+1 / 2)^{2}}}\right]\right\} \\
& \simeq-\left|\beta+\frac{1}{2}\right|\left[1+\ln \left(\frac{\left|k \eta_{\mathrm{f}}\right|}{2|\beta+1 / 2|}\right)\right],
\end{aligned}
$$

where in the last expression we have considered that $\left|k \eta_{\mathrm{f}}\right|$ $\ll 1$. Inserting this result in Eqs. (43) and (44), one finds

$$
\begin{gathered}
\mathcal{P}_{\zeta}=\frac{\ell_{\mathrm{Pl}}^{2}}{\ell_{0}^{2}} \frac{1}{\pi \epsilon_{1}} g(\beta) k^{2 \beta+4}, \quad \mathcal{P}_{h}=\frac{\ell_{\mathrm{Pl}}^{2}}{\ell_{0}^{2}} \frac{16}{\pi} g(\beta) k^{2 \beta+4}, \\
g(\beta) \equiv \frac{2 e^{2 \beta+1}}{(2 \beta+1)^{2 \beta+2}} .
\end{gathered}
$$

These expressions are the main result of this section. They should be compared to the exact result given in Eqs. (49) and (50). As expected, they are time-independent.

The first observation is that the shape of the power spectra is exactly reproduced by the WKB approximation. In particular, this means that the spectral indices and their runnings (as well as the runnings of the runnings, and so on) are predicted exactly by the WKB approximation. The amplitude is given by the function $g(\beta)$, whereas in the exact case, it is given by the function $f(\beta)$; see Eq. (51). We have $g(\beta=$ $-2)=18 \times \mathrm{e}^{-3} \simeq 0.896$, a value that should be compared with $f(\beta=-2)=1$. In the limit where $|\beta|$ goes to infinity we can use the Stirling formula, $\Gamma(x) \simeq \sqrt{2 \pi} x^{x-1 / 2} \mathrm{e}^{-x}$ to approximate $f(\beta)$ and one finds that $\lim _{\beta \rightarrow-\infty} f(\beta)$ $\rightarrow g(\beta)$, i.e. the WKB approximation gives the exact result in this limit. It is interesting to notice that this limit corresponds to going away from scale invariance, i.e. to "fastroll" inflationary models (the kinetic energy of the inflaton is of the same order as its potential energy). Therefore, the power-law inflation with $|\beta| \gg 2$ is an explicit example of a model where the WKB approximation gives an accurate result in a regime where the slow-roll approximation cannot be used to compute the power spectrum.

Another remark is in order. The fact that the WKB ap- 


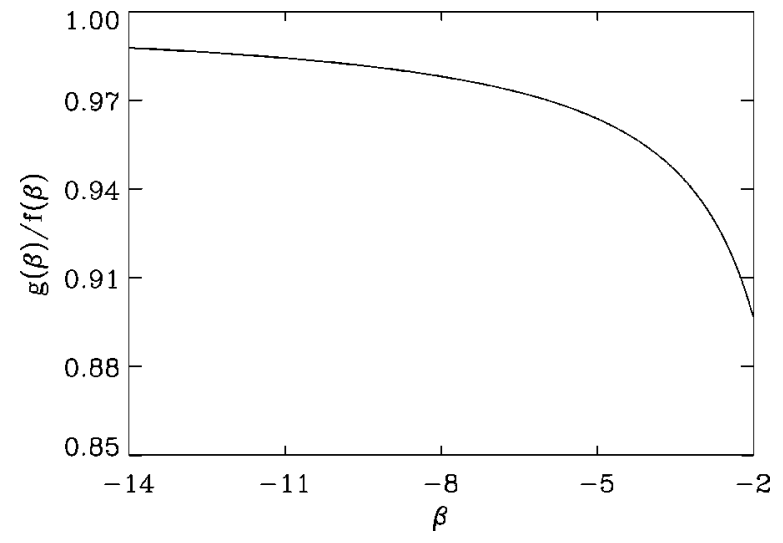

FIG. 1. Evolution of the ratio $g(\beta) / f(\beta)$ with $\beta$. In the limit $|\beta| \rightarrow \infty$, the ratio tends towards 1 . This can only be guessed from the figure because the convergence rate is slow.

proximation is better for large values of $|\beta|$ than for small values of $|\beta+2|$ (scale invariance) might seem to be in contradiction with Eqs. (27) and (28), which indicate that $\left|Q / \omega^{2}\right|$ decreases as the slow-roll parameters approach zero (scale invariance). In fact, there is no contradiction because the WKB criterion only measures the accuracy with which the mode function is predicted at a given time, without taking into account the error coming from the matching procedure, while the error in the power spectrum is a combination of these two sources of error.

The conclusion is that the WKB amplitude of the spectrum is always a good approximation, see Fig. 1, although the precision of the prediction for the amplitudes in the case of almost scale-invariant inflation is not as good as in the slow-roll approximation [1,13-15]. Contrary to the latter, the spectral indices are predicted exactly by the WKB approximation, $n_{\mathrm{S}}-1=n_{\mathrm{T}}=2 \beta+4$. This is a big advantage if data sets of large dynamic ranges are investigated.

\section{APPLICATION TO SLOW-ROLL INFLATION}

Let us now compare the WKB approximation with the (leading-order) slow-roll approximation. A comment is in order here on the meaning of the phrases "slow-roll conditions" and "slow-roll approximation." If the slow-roll conditions hold, all slow-roll parameters are small. This does not specify the approximate scheme that one should use in order to calculate the power spectra; in particular it does not imply that the slow-roll approximation has to be utilized in this situation. In this section, we will determine the power spectra computed with the help of the WKB approximation in a situation where the slow-roll conditions are valid. Having clarified this point, let us first quote the result of the slow-roll approximation $[13,14]$ :

$$
\begin{aligned}
\mathcal{P}_{\zeta}(k)= & \frac{H^{2}}{\pi \epsilon_{1} m_{\mathrm{Pl}}^{2}}\left[1-2(C+1) \epsilon_{1}-C \epsilon_{2}-\left(2 \epsilon_{1}+\epsilon_{2}\right) \ln \left(\frac{k}{k_{*}}\right)\right. \\
& \left.+\mathcal{O}\left(\epsilon_{n}^{2}\right)\right]
\end{aligned}
$$

$$
\mathcal{P}_{h}(k)=\frac{16 H^{2}}{\pi m_{\mathrm{Pl}}^{2}}\left[1-2(C+1) \epsilon_{1}-2 \epsilon_{1} \ln \left(\frac{k}{k_{*}}\right)+\mathcal{O}\left(\epsilon_{n}^{2}\right)\right],
$$

where $C \equiv \gamma_{\mathrm{E}}+\ln 2-2 \simeq-0.7296, \gamma_{\mathrm{E}} \simeq 0.5772$ being the Euler constant. All quantities are evaluated at $\eta_{*}$, which is the moment of time when $k_{*}=a H\left(N_{*}\right)$. The scale $k_{*}$ is called the pivot scale. It has been shown in Ref. [14] that the corresponding time $\eta_{*}$ has a nice interpretation: it is nothing but the time at which the matching between the region where $a H \simeq k$ and the superhorizon region is performed. It is important to realize that, according to the previous definition, $\eta_{*}$ is not a function of $k$, i.e. is the same for all scales. Changing the time of matching is therefore equivalent to changing the pivot scale. This means that the pivot scale must be in the range of scales that are of astrophysical interest today but otherwise can be chosen freely. It has been demonstrated in Ref. [14] that the location of the pivot scale can be optimized to reduce the slow-roll error in the power spectrum.

From Eqs. (59) and (60), we notice that close to the de Sitter inflation the slow-roll approximation predicts the amplitude for both density and tensor perturbations very well. The accuracy of the slow-roll approximation improves as one approaches scale invariance, since this corresponds to $\epsilon_{n} \rightarrow 0$ (for power-law inflation this corresponds to $\beta \rightarrow$ $-2)$. A priori, it gives an exact result for exact scale invariance but then the linear approximation blows up, as can be seen from Eq. (59), when $\epsilon \rightarrow 0$.

In contrast, the WKB approximation does not give an exact result for the amplitude in the scale-invariant limit (see the discussion of the limit $\beta \rightarrow-2$ above), but it is able to give a reasonable approximation in the regime where slowroll is violated $\left(\epsilon_{1} \rightarrow 1\right)$. The spectral indices predicted by the slow-roll approximation at first order are

$$
n_{\mathrm{S}}-1=-2 \epsilon_{1}-\epsilon_{2}, \quad n_{\mathrm{T}}=-2 \epsilon_{1} \text {. }
$$

These estimates are not exact, contrary to the WKB result in the case of power-law inflation, but provide good estimates close to the scale-invariant situation.

Let us now evaluate the WKB power spectra for the situation of slow-roll inflation. For that purpose we expand Eqs. (43) and (44) at first order in the horizon flow functions and compare the result with Eqs. (59) and (60). The effective frequencies read

$$
\begin{aligned}
& \omega_{\mathrm{S}}^{2}=k^{2} \eta^{2}-\left(\frac{9}{4}+3 \epsilon_{1}+\frac{3}{2} \epsilon_{2}\right), \\
& \omega_{\mathrm{T}}^{2}=k^{2} \eta^{2}-\left(\frac{9}{4}+3 \epsilon_{1}\right),
\end{aligned}
$$

and the WKB power spectra become

$$
\mathcal{P}_{\zeta}=\frac{H^{2}}{\pi \epsilon_{1} m_{\mathrm{Pl}}^{2}}\left(-k \eta_{\mathrm{f}}\right)^{3} \frac{2}{3}\left(1-\frac{8}{3} \epsilon_{1}-\frac{1}{3} \epsilon_{2}\right) \mathrm{e}^{2 \Psi_{\mathrm{s}}}
$$




$$
\mathcal{P}_{h}=\frac{16 H^{2}}{\pi m_{\mathrm{Pl}}^{2}}\left(-k \eta_{\mathrm{f}}\right)^{3} \frac{2}{3}\left(1-\frac{8}{3} \epsilon_{1}\right) \mathrm{e}^{2 \Psi_{\mathrm{T}}}
$$

where we have made use of the relation $1 /\left[\left(1-\epsilon_{1}\right) a H\right]=$ $-\eta\left[1+\mathcal{O}\left(\epsilon_{n}^{2}\right)\right]$. The evaluation of the integrals in the argument of the exponential functions gives

$$
\begin{gathered}
\Psi\left(\eta_{\mathrm{f}}, \eta_{*} ; k\right)=\int_{x_{*}}^{x_{f}}|\omega(y ; k)| \mathrm{d} y \\
=-\int_{\eta_{*}(b)}^{\eta_{\mathrm{f}}} \sqrt{\frac{9}{4}-k^{2} \tau^{2}+b(\epsilon, \delta, \xi)} \frac{\mathrm{d} \tau}{\tau},
\end{gathered}
$$

with

$$
\begin{aligned}
& b_{\mathrm{S}}\left(\epsilon_{n}\right) \equiv 3 \epsilon_{1}+\frac{3}{2} \epsilon_{2}, \quad b_{\mathrm{T}}\left(\epsilon_{n}\right) \equiv 3 \epsilon_{1}, \\
& \eta_{*}(b)=-\frac{1}{k} \sqrt{\frac{9}{4}+b\left(\epsilon_{n}\right) .}
\end{aligned}
$$

At first order, we may consider the horizon flow functions as being constants. This is due to the fact that the derivatives of the horizon flow functions are quadratic in these parameters. The integration can be performed as for power-law inflation in the previous section. The final result is

$$
\begin{aligned}
\mathcal{P}_{\zeta}(k)= & \frac{H^{2}}{\pi \epsilon_{1} m_{\mathrm{Pl}}^{2}}\left(18 \mathrm{e}^{-3}\right)\left[1-2(D+1) \epsilon_{1}-D \epsilon_{2}\right. \\
& \left.-\left(2 \epsilon_{1}+\epsilon_{2}\right) \ln \left(-k \eta_{\mathrm{f}}\right)+\mathcal{O}\left(\epsilon_{n}^{2}\right)\right], \\
\mathcal{P}_{h}(k)= & \frac{16 H^{2}}{\pi m_{\mathrm{Pl}}^{2}}\left(18 \mathrm{e}^{-3}\right)\left[1-2(D+1) \epsilon_{1}\right. \\
& \left.-2 \epsilon_{1} \ln \left(-k \eta_{\mathrm{f}}\right)+\mathcal{O}\left(\epsilon_{n}^{2}\right)\right],
\end{aligned}
$$

with $D \equiv \frac{1}{3}-\ln 3 \approx-0.765$, a reasonable approximation of $C$ from above. All the quantities in the above equation are evaluated at $\eta=\eta_{\mathrm{f}}$. In order to compare this result with the slow-roll expressions (59) and (60) we have to establish a link between $H\left(N_{\mathrm{f}}\right)$ and $H\left(N_{*}\right), \epsilon_{1}\left(N_{\mathrm{f}}\right)$ and $\epsilon_{1}\left(N_{*}\right)$, and so on. At the level of a first-order expansion in the horizon flow functions, we can consider $\epsilon_{1}$ and $\epsilon_{2}$ to be constant everywhere, except in the factor $1 / \epsilon_{1}$ in Eq. (59). Performing a linear expansion of $\epsilon_{1}$ around $N_{*}$ we find

$$
\frac{1}{\epsilon_{1}\left(N_{\mathrm{f}}\right)}=\frac{1}{\epsilon_{1}\left(N_{*}\right)}\left[1-\epsilon_{2}\left(N_{*}\right) \Delta N+\cdots\right] .
$$

In a similar manner we find

$$
H^{2}\left(N_{\mathrm{f}}\right)=H^{2}\left(N_{*}\right)\left[1-2 \epsilon_{1}\left(N_{*}\right) \Delta N+\cdots\right] .
$$

We eliminate $-\eta_{\mathrm{f}}$ in the logarithm by expressing it in terms of $1 /(a H)\left(N_{\mathrm{f}}\right)$. Corrections containing $\epsilon_{1}$ inside the logarithm can be omitted since they contribute at higher order only. Finally, the logarithm can be written

$$
\begin{aligned}
\ln \left(-k \eta_{\mathrm{f}}\right) & =\ln \left[\frac{k}{(a H)\left(N_{*}\right)}\right]+\ln \left[\frac{(a H)\left(N_{*}\right)}{(a H)\left(N_{\mathrm{f}}\right)}\right]+\cdots \\
& =\ln \left(\frac{k}{k_{*}}\right)-\Delta N+\cdots,
\end{aligned}
$$

where now the dots denote terms of order $\epsilon_{1}$ and we have used the pivot scale $k_{*}=(a H)\left(N_{*}\right)$. Putting everything together we find that all $\Delta N$ cancel, and the result simply reads

$$
\begin{aligned}
\mathcal{P}_{\zeta}(k)= & \frac{H^{2}}{\pi \epsilon_{1} m_{\mathrm{Pl}}^{2}}\left(18 \mathrm{e}^{-3}\right)\left[1-2(D+1) \epsilon_{1}-D \epsilon_{2}-\left(2 \epsilon_{1}\right.\right. \\
& \left.\left.+\epsilon_{2}\right) \ln \left(\frac{k}{k_{*}}\right)+\mathcal{O}\left(\epsilon_{n}^{2}\right)\right] \\
\mathcal{P}_{h}(k)= & \frac{16 H^{2}}{\pi m_{\mathrm{Pl}}^{2}}\left(18 \mathrm{e}^{-3}\right)\left[1-2(D+1) \epsilon_{1}-2 \epsilon_{1} \ln \left(\frac{k}{k_{*}}\right)\right. \\
& \left.+\mathcal{O}\left(\epsilon_{n}^{2}\right)\right]
\end{aligned}
$$

which can now be compared with the prediction from the slow-roll power spectra (59) and (60).

It is seen immediately that the spectral indices coincide with the slow-roll result (61). As already expected from the discussion of power-law inflation above, the spectral indices are predicted by the WKB approximation very well, the amplitudes only at an accuracy of $10 \%$ (coming from the factor $18 \mathrm{e}^{-3}$ ). This is certainly not good enough for future experiments, but it is well known that WKB approximations can be systematically improved.

\section{CONCLUSIONS}

A new method to predict inflationary power spectra has been presented, based on the WKB approximation. One of the main advantages of this new scheme of approximation is that it can give a good approximation even if the slow-roll conditions are violated, whereas, up to now, all of the methods in the literature were subject to this limitation [16]. We have checked this fact explicitly on the example of powerlaw inflation. In addition, we have also tested the method for the case of slow-roll inflation and find errors below $10 \%$ for all quantities considered. The prediction of the spectral indices is exact in the case of the power-law inflation. Assuming that the conclusions obtained from the power-law case are also valid in a more general context, the WKB approximation appears as an efficient method, especially for predicting the spectral indices. The main restriction comes from the amplitude, which is not predicted with a similar accuracy as the slow-roll approximation close to scale invariance.

A possible disadvantage of the method is that it is nonlocal in the sense that an integration is involved from the time of horizon crossing until some time when all the modes of interest are on superhorizon scales. This means, in contrast to the slow-roll approximation, where a certain behavior of the background is assumed, that one needs to know how the Hubble flow functions evolve with time in principle. In 
the example of slow-roll inflation it has been shown that one essential step in the WKB approximation is to link the predictions at some time $\eta_{\mathrm{f}}$ to the parameters relevant at the time of horizon crossing.

Many improvements of the results presented in this article are possible. As already mentioned above, the WKB expansion can be pushed further, e.g. by going beyond the linear approximation for the joining of the super- and subhorizon WKB solutions. This will reduce the error in the amplitude, therefore avoiding the main shortcoming of the new method. Another point is that the standard slow-roll approximation (Bessel approximation) is valid only when the slow-roll parameters are constant. Therefore, it cannot be pushed beyond the first order. Recently, Stewart and Gong [15] have proposed a method, based on Green functions perturbative calculations, which is free from this problem. They have calculated the inflationary power spectra up to the second order. The WKB approximation is also free from the above limitation and therefore allows a determination of the spectra beyond the leading order. All these questions are currently under investigation [17].
Note added. While the present article was being completed, a new paper [18] appeared where the inflationary power spectra are also calculated using a new method of approximation, namely the "uniform approximation" according to the terminology used in that article. Some of the equations obtained in [18] bear a close resemblance to the results obtained in the present work as revealed by a comparison of Eqs. (43) and (44) with Eq. (14) of Ref. [18]. After having released the first version of this paper, we became aware of an earlier attempt to use the WKB approximation for cosmological perturbations by Nagasawa and Yokoyama [19].

\section{ACKNOWLEDGMENTS}

We thank F. Finelli, M. Nagasawa, C. Terrero-Escalante and G. Venturi for useful comments on the first version and we acknowledge support from the bilateral Austrian-French AMADEE program.
[1] S.M. Leach, A.R. Liddle, J. Martin, and D.J. Schwarz, Phys. Rev. D 66, 023515 (2002).

[2] L.P. Grishchuk, Zh. Eksp. Teor. Fiz. 67, 835 (1974) [Sov. Phys. JETP 40, 409 (1974)]; V.F. Mukhanov, Pis'ma Zh. Eksp. Teor. Fiz. 41, 402 (1985) [JETP Lett. 41, 493 (1985)]; Zh. Eksp. Teor. Fiz. 84, 1 (1988) [Sov. Phys. JETP 67, 1297 (1988)]; V.F. Mukhanov, H.A. Feldman, and R.H. Brandenberger, Phys. Rep. 215, 203 (1992).

[3] H.A. Kramers, Z. Phys. 39, 836 (1926).

[4] L.A. Young and G.E. Uhlenbeck, Phys. Rev. 36, 1158 (1930).

[5] R.E. Langer, Phys. Rev. 51, 669 (1937).

[6] J. M. Bardeen, in Particle Physics and Cosmology, edited by A. Zee (Gordon \& Breach, New York, 1989).

[7] M.B. Hoffman and M.S. Turner, Phys. Rev. D 64, 023506 (2001).

[8] D.J. Schwarz, C.A. Terrero-Escalante, and A.A. Garcia, Phys. Lett. B 517, 243 (2001).

[9] L. Wang, V.F. Mukhanov, and P.J. Steinhardt, Phys. Lett. B
414, 18 (1997).

[10] L.F. Abbott and M.B. Wise, Nucl. Phys. B244, 541 (1984).

[11] J. Martin and D.J. Schwarz, Phys. Rev. D 57, 3302 (1998).

[12] I. S. Gradshteyn and I. M. Ryzhik, Tables of Integrals, Series and Products (Academic, New York, 1981).

[13] E.D. Stewart and D.H. Lyth, Phys. Lett. B 302, 171 (1993).

[14] J. Martin and D.J. Schwarz, Phys. Rev. D 62, 103520 (2000); J. Martin, A. Riazuelo, and D.J. Schwarz, Astrophys. J. Lett. 543, L99 (2000).

[15] E.D. Stewart and J.O. Gong, Phys. Lett. B 510, 1 (2001).

[16] Exceptions are the constant and growing horizon approximations of Ref. [8]. These approximations relax the slow-roll conditions by allowing that one of the horizon flow functions is much larger than all the others, but still smaller than unity.

[17] J. Martin and D. J. Schwarz (in preparation).

[18] S. Habib, K. Heitmann, G. Jungman, and C. Molina-Paris, Phys. Rev. Lett. 89, 281301 (2002).

[19] M. Nagasawa and J. Yokoyama, Nucl. Phys. B370, 472 (1992). 